Bangladesh J. Plant Taxon. 17(2): 141-165, 2010 (December)

(C) 2010 Bangladesh Association of Plant Taxonomists

\title{
PLANT COLLECTIONS FROM BANGLADESH IN THE HERBARIUM AT SHILLONG (ASSAM), INDIA
}

\author{
Hussain Ahmed Barbhuiya And R. Gogol ${ }^{1}$ \\ Botanical Survey of India, Eastern Regional Centre, Shillong, India
}

Keywords: Bangladesh; Plant collections; Assam.

\begin{abstract}
The paper presents a complete list of plants from Bangladesh deposited in the herbarium of the Botanical Survey of India at Shillong (ASSAM). A total of 448 species belonging to 324 genera and 101 families has been found to be deposited in the herbarium in Assam. Detailed field data of each species have also been provided.
\end{abstract}

\section{Introduction}

The study on the flora of North East India was actually initiated by Gustav Mann, who was appointed as the in-charge of the rubber plantation in Chariduar in the year 1863 and later became the first Conservator of Forests of Assam. During his service he had to collect a good number of plants for his higher authority Dietrich Brandis, the first Inspector General of Forest, from the Assam province which in those days also included the district of Sylhet, presently in Bangladesh. Mann sent one set of his collections to Brandis and kept a duplicate set at Shillong, the Headquater of Assam forest department for future reference. In this way Mann led the foundation of a forest herbarium at Shillong. During the period of Mann, Tara Kisor Gupta and Boloram Sing also deposited their collections in the forest herbarium at Shillong. Their excellent contributions also helped contemporary botanists to understand the flora of Sylhet. Later in 1912, A. Earle, the then Chief Commissioner of Assam, sanctioned a huge project entitled 'Flora of Assam', to Upendra Nath Kanjilal the then Extra Assistant Conservator of Forests of Assam. U.N. Kanjilal, a veteran field botanist started collecting plants form different parts of the then Assam province. As a result of his collection a good number of plants also collected from Sylhet (Sylhet was under Assam Province) were deposited at the forest herbarium at Shillong. When U. N. Kanjilal suddenly died in 1928 before completion of 'Flora of Assam', the charge of the project was handed over to his capable son P. C. Kanjilal a forest officer from U.P. cadre. Along with P. C. Kanjilal many other foresters like A. Das, C. S. Purkayastha, R. N. De, B. C. Sengupta, G. K. Deka, N. L. Bor, B. B. Shyam, S. R. Sharma, Dinanath Paul and H. K. Dastider joined hands to enrich the collection in the forest herbarium from Sylhet and its adjoining areas.

${ }^{1}$ Corresponding author. Botanical Survey of India, Arunachal Pradesh Regional Centre, Itanagar, India. E-mail: rajibbsi@yahoo.co.in 
The collections made by these pioneer workers helped immensely to publish the 'Flora of Assam' (1934-1940) which is a monumental floristic work. It helps to know the regional botany of the north eastern part of the Indian subcontinent. After the publication

of the 'Flora of Assam, few people from Assam forest department continued collecting plants from Sylhet and adjoining areas. Some of them are R. N. De, G. K. Deka and M. M. Srinivasan.

In the year 1956, the Botanical Survey of India established a regional office at Shillong and its herbarium is known by the acronym ASSAM (Holmgren et al., 1990). This Herbarium, the largest Herbarium of the region, received all the 45,000 herbarium specimens from the forest Herbarium which was long nonfunctional after the publication of the 'Flora of Assam'. In this way all the plants that were collected from various places of Assam province, got deposited at ASSAM.

In course of the study at ASSAM the authors documented the plants from Sylhet, Chittagong and neighbouring areas in Bangladesh. It gives a clear picture about the floristic wealth of these areas. In fact when Kanjilal et al. (1934-1940) published the 'Flora of Assam' all the species collected up to that time by their colleagues and earlier workers from different parts of the present day Bangladesh, were included in their book but the details of the collections were not cited. In the present checklist of plants, all the species from Bangladesh deposited so far at ASSAM have been taken into account along with the details of their collections. This will be helpful to the foresters, field botanists, conservationists and researchers to relocate the plants or to judge their present status in their earlier site of collections which were carried out more than half century ago and to formulate conservational strategies to protect these national resources.

\section{Material and Methods}

A total number of 2,65,000 herbarium sheets were screened at ASSAM to find out all the collections from Bangladesh. The botanical names were recorded with the place of collection, collectors' names, collection numbers with particular date or the month of the year in which they have been collected. The families have been arranged in the list according to Bentham and Hooker's system of classification (Batham and Hooker, 18621883) with modifications followed at Kew and British Museum. The accession numbers of the sheets have only been given after s.n. (sine numero) i.e. when there was no collector's number. The correct names of the species have been given by consulting the latest literature. For the three species of Dendrocalamus the names of the species could not be ascertained and in these cases, their local names, as recorded on the herbarium sheets, have been given in brakets against them. 


\section{Result and Discussion}

During the screening of the herbarium specimens, a total number of 448 species under 324 genera and 101 families were found to be deposited at ASSAM from present Bangladesh area, out of which 350 species belonging to 252 genera and 87 families were dicots and 98 species belonging to 72 genera and 14 families were monocots. Among the dicots Fabaceae was the largest family with 22 species belonging to 15 genera. On the other hand, Poaceae was the largest family among the monocots with 48 species belonging to 35 genera. Among the collectors U. N. Kanjilal made the largest number of collections with 111 species and G. K. Deka made the second largest collection with 109 species. Apart from the aforesaid names of collectors, plant collections of J. S. Gamble and C. B. Clarke from Bangladesh were also found to be deposited at ASSAM. Further some more collections from Chittagong, made by Rev. W. J. L. Wenger, Christian missionary posted for medical relief work in Mizoram and neighbouring areas in the first few decads of $20^{\text {th }}$ century were found to be deposited at ASSAM.

\section{Enumeration of species}

\section{DICOTYLEDONS}

\section{Ranunculaceae}

Clematis cadmia Buch-Ham ex Hook. f. \& Thomson; Sylhet, April 1877, G. Mann s.n. 41632.

Naravelia zeylanica (L.)DC.; Chhatak, Sylhet, 21.11.1943, G.K. Deka 21795; Sylhet, October 1936, C.S. Purkayastha 12439.

Ranunculus sceleratus L.; Chhatak, 7.02.1941, Sylhet, G.K. Deka 20285.

\section{Dilleniaceae}

Tetracera scandens (L.) Merr.; Longai, Sylhet, 24.12.1914, U. N. Kanjilal 4924.

\section{Magnoliaceae}

Kadsura heteroclita (Roxb.) Craib.; Tillagarh, Sylhet, 14.11.1932, A. Das 10438.

Magnolia pterocarpa Roxb.; Near Jaintiapur, Sylhet, March 1890, G. Mann s. n. 201.

Michelia champaca L.; Dohalia Reserve, Sylhet, 28.07.1932, P. C. Kanjilal s.n. 335; Jaintia Hills, Sylhet, November 1889, Boloram Sing s.n. 338; near Bisnuri, Sylhet, 30.04.1915, U. N. Kanjilal 5088.

M. mannii King; Longai, Sylhet district, August 1890, U. N. Kanjilal 343.

\section{Annonaceae}

Fissistigma rubiginosum (DC.) Merr.; Chandkhira T. E. Sylhet, 29.12.1914, U. N. Kanjilal 4956.

Polyalthia simiarum (Buch.-Ham. ex Hook. f. \& Thompson) Hook. f. \& Thompson; 
Longai Reserve, Sylhet, 28.12.1914, U. N. Kanjilal 4946.

\section{Menispermaceae}

Cissampelos pareira L.; Lawachara, Sylhet, 18.8.1938, R. N. De 18128.

\section{Nympheaceae}

Nymphaea pubescens Willd.; Sylhet, October 1935, C. S. Purkayastha 12451.

\section{Capparidaceae}

Capparis zeylanica L.; Teliapara, Sylhet, 12.4.1947, M. M. Srinivasan s. n. 39000.

Cleome spinosa Jacq.; Sylhet, March 188, G. Mann s. n. 1058.

C. viscosa L.; Sylhet, April 1881, G. Mann 34.

Strixis suaveolens (Roxb) Pierre; Lawachara, Sylhet, 21.2.1941, R. N. De 20530.

\section{Violaceae}

Viola betonicifolia Sm.; Sylhet, April 1877, G. Mann s. n. 1188.

\section{Flacourtiaceae}

Hydnocarpus kurzii (King.) Warb.; Lawachara, Sylhet, 15.4.1940, R. N. De 19329; Longai Reserve, Sylhet, 28.12.1914, U.N. Kanjilal 4949.

\section{Samydaceae}

Homalium bhamoense Cubitt. \& W. W. Sm.; Sylhet, May 1937, N. L. Bor 13929; Silua forest, 21.3.1921, U. N. Kanjilal 7639; Silua forest, Sylhet, 29.3.1921, B. C. Sengupta 7639 .

H. schlichii Kurz; Badshai Tilla, Sylhet, 16.011932, U. N. Kanjilal 7726.

\section{Polygalaceae}

Securidaca inappendiculata Hassk; Jaintiapur, 7.11.1936, Sylhet, G. K. Deka 16234.

Xanthophyllum flavescens Roxb.; Rajkandi, Sylhet, March 1935, C. S. Purkayastha 10988.

\section{Caryophyllaceae}

Polycarpon prostratum (Forsk) Asch. \& Schweinf.; Chhatak, Sylhet, 7.2.1941, G. K. Deka 22218.

Stellaria media (L.) Vill.; Longai, Sylhet, 15.2.1941, R. N. De 20432.

\section{Clusiaceae}

Calophyllum polyanthum Wall. ex Choisy; Singla Reserve, Sylhet, 15.3.1921, U. N. Kanjilal 7634.

Garcinia kydia Roxb.; Longai Reserve, Sylhet, 28.3.1932, P. C. Kanjilal 10123; Longai Reserve, Sylhet, 1.6.1932, P. C. Kanjilal 10245. 
G. pedunculata Roxb.; Sylhet, 8.3.1947, D. Paul 22090.

G. pictoria (Roxb.) Engler; Singla Reserve, Sylhet, 22.12.1914, U. N. Kanjilal 4911.

G. sopsopia (Buch.-Ham) Mabb.; Longai Reserve, Sylhet, 24.12.1914, U. N. Kanjilal 4922.

Mesua ferrea L.; Sylhet, March 1887, T.K. Gupta s. n. 1970.

M. floribunda (Wall.) Kosterm.; Patharia Forest, Sylhet, July 1854, T. K. Gupta s.n. 1913; Loobah Reserve, Sylhet, 10.11.1914, U. N. Kanjilal 4698; Singla Reserve, Sylhet, 13.3.1921, U. N. Kanjilal 7635.

\section{Theaceae}

Camellia kissi Wall.; Longai Reserve, Sylhet, 26.12.1914, U. N. Kanjilal 4931.

Eurya acuminata D.C.; Lawachara, Sylhet, 14.9.1946, G. K. Deka 21994.

Schima wallichii Choisy; Patharia forest, Sylhet, March 1886, T. K. Gupta s. n. 2240.

\section{Dipterocarpaceae}

Dipterocarpus turbinatus Gaertn. f.; Patharia forest, Sylhet, March 1886, G. Mann s.n. 2404; Longai Reserve, Sylhet, 28.11.1931, P. C. Kanjilal 9817; Patharia Reserve, Sylhet, May 1935, R. D. Silwa 11087.

\section{Malvaceae}

Hibiscus macrophyllus Roxb. ex Hornem.; Longai Reserve, Sylhet, March 1919, B. Sen Gupta 60.

Kydia calycina Roxb.; Patharia forest, Sylhet, March 1886, G. Mann s. n., 2638.

Thespesia lampas (Cav.) Dalz. ex Dalz \& A. Gibson; Raghunandan Reserve, Sylhet, 16.1.1925, U. N. Kanilal 7835.

\section{Sterculiaceae}

Buettneria pilosa Roxb.; Lawachara, Sylhet, 19.12.1938, R. N. De 17727; Sylhet, October 1935, C. S. Purkayastha 12433.

Heritiera macrophylla Wall. ex Voigt; Jaintiapur, Sylhet, 7.11.1936, G. K. Deka 162050.

H. papilio Bedd.; Longai Reserve, Sylhet, 27.12.1914, U. N. Kanjilal 4934.

Melochia corchorifolia L.; Haluganj, Sylhet, October 1935, C. S Purkyastha 12440.

Pterospermum lanceaefolium Roxb.; Naricha Lakhimamla, Sylhet, March 1886, T. K. Gupta s.n. 2849.

Sterculia guttata Roxb.; Singla Reserve, Sylhet, 22.12.1914, U. N. Kanjilal 4908.

S. roxburghii Wall.; Sylhet, March 1886, G. Mann s.n. 2728.

S. villosa Roxb.; Patharia forest, Sylhet, March 1886, T. K. Gupta 32. 


\section{Tiliaceae}

Grewia piscatorum Hance; Sylhet, October 1935, C. S. Purkayastha 12449.

G. serrulata DC.; Lawachara, Sylhet, 19.8.1938, R. N. De 19056.

Microcos paniculata L.; Lawachara, Sylhet, 10.8.1938, G. K. Deka 18125.

Triumfetta rhomboidea Jacq.; Bhanugach, Sylhet, 14.11.1932, A. Das 10437.

\section{Elaeocarpaceae}

Elaeocarpus floribundus Blume; Santgaon village, Sylhet, 10.11.1914, U. N. Kanjilal 4702; Sylhet town, 4.9.1941, R. N. De 20602.

Sloanea sterculiacea (Benth.) Rehder \& E.H. Wilson; Patharia forest, Sylhet, March 1886, G. Mann s.n. 3086.

\section{Linaceae}

Erythroxylum coca Lam.; Chandkhera Tea Estate, Sylhet, R. N. De 19155.

Ixonanthes reticulata Jack; Longai Reserve, Sylhet, 27.05.1937, N. L. Bor 16077; Longai Reserve, Sylhet, 24.12.1914, U. N. Kanjilal 4926.

Linum usitatissimum L.; Chhatak, Sylhet, 7.2.1941, G. K. Deka 20288.

\section{Malpighiaceae}

Aspidopterys indica (Roxb.) Hochr.; Sylhet, January 1936, C. S. Purkayastha 13445.

\section{Rutaceae}

Acronychia pedunculata (L.) Miq.; Bhanugach Reserve, Sylhet, 12.8.1921, U. N. Kanjilal 7639.

Citrus assamensis S. Dutta \& S. C. Bhattacharya; Sylhet, 6.11.1938, S. Dutta 23059.

C. latipes (Swingle) Tanaka; Sylhet, 20.2.1936, D. Paul 13600.

Clausena anisata (Willd.) Hook. f. ex Benth. var. paucijuga (Kurz) J. F. Molino; Raghunandan Reserve, Sylhet, 12.4.1947, M. M. Srinivasan 22239.

C. macroptera Montrouz.; Dawki, Sylhet, 31.5.1935, G. K. Deka 12204.

\section{Ochnaceae}

Ochna integerrima (Lour.) Merr.; Tilagarh Reserve, Sylhet, June 1935, C. S. Purekayastha 10970.

\section{Burseraceae}

Bursera serrata Wall. ex Coleber; Sylhet, January 1936, C. S. Purkayastha 13435.

Garuga floribunda Decne var. gamblei (King ex Smith) Kalkman; Near Baithakhal Tea Estate, Sylhet, 29.12.1914, U. N. Kanjilal 4955. 


\section{Meliaceae}

Aglaia perviridis Hiern; Singla Reserve, Sylhet, 15.1.1932, U. N. Kanjilal 7725.

Dysoxylum binectariferum (Roxb.) Hook. f. ex Bedd.; Longai Reserve, Sylhet, 26.12.1914, U. N. Kanjilal 4930.

Cedrela microcarpa (C. DC.) Harms; Longai Reserve, Sylhet, 17.3.1915, U. N. Kanjilal 6901.

Walsura robusta Roxb.; Longai Reserve, Sylhet, 20.3.1921, U. N. Kanjilal 7640.

\section{Olacaceae}

Natsiatum herpeticum Buch.-Ham. ex Arn.; Bholaganj forest, Sylhet, 23.01.1931, P. C. Kanjilal 9001; Cheragi Path, Sylhet, 23.12.1914, U. N. Kanjilal 4918.

\section{Celastraceae}

Bhesa robusta (Roxb.) Ding-Hou.; Sylhet, January 1936, C. S. Purkayastha 13432.

Celastrus stylosus Wall.; Sylhet, 1.12.1910, U. N. Kanjilal s.n. 5553.

Euonymus attenuatus Wall. ex Lawson; Loobah Lake, Sylhet, 9.11.1914, U. N. Kanjilal 4687.

Lophopetalum wightianum Arn.; Ichabil tea garden, Sylhet, 28.12.1914, U. N. Kanjilal 4951; Longai Reserve, Sylhet, 18.3.1915, U. N. Kanjilal 6902.

\section{Rhamnaceae}

Ziziphus oenoplia (L.) Mill.; Longai forest, Sylhet, 16.2.1941, R. N. De 20606.

\section{Vitaceae}

Ampelocissus barbata (Wall.) Planch.; Lawachara, Sylhet, 19.8.1938, R. N. De 18071.

Cissus adnata Roxb.; Chhatak, Sylhet, 9.2.1941, G. K. Deka 20541; Loobah, Sylhet, 8.11.1914, U. N. Kanjilal 4679.

C. repens Lam.; Sylhet Division, October 1935, C. S. Purkayastha 12431.

Tetrastigma dubium (Lawson) Planch.; Chhatak, Sylhet, 9.2.1941, G. K. Deka 20542.

T. rumicispermum. (Lawson) Planch.; Patharia forest, Sylhet, July 1828, G. Mann 1073.

\section{Sapindaceae}

Aesculus assamicus Griff.; Patharia forest, Sylhet, March 1886, T. K. Gupta s. n. 6346.

Allophylus cobbe Blume; Lawachara, Sylhet, 18.8.1938, G. K. Deka 18126.

Erioglossum rubiginosum (Roxb.) Blume; Jaintiapur, Sylhet, May 1937, N. L. Bor 13944; Patheria, Sylhet, May 1884, T.K. Gupta s.n. 6297.

Lepisanthes senegalensis (A. Juss. ex Poir.) Leenh.; Tillagarh Reserve, Sylhet, 9.4.1947, M. M. Srinivasan 22343. 


\section{Sabiaceae}

Meliosma simplicifolia (Roxb.) Walp.; Chhatak, Sylhet, 7.2.1941, G. K. Deka 20497.

\section{Anacardiaceae}

Holigarna longifolia Buch.-Ham. ex Roxb.; Longai Reserve, Sylhet, March 1935, C. S. Purkayastha 10987; Sylhet, June 1920, U. N. Kanjilal 7832.

Mangifera indica L.; Longai, Sylhet, 18.2.1941, R. N. De 20520.

Pegia nitida Colebr.; Longai forest, Sylhet, 16.2.1941, R. N. De 20565.

Semecarpus prainii King.; Patharia Reserve, Sylhet, 10.3.1920, U. N. Kanjilal 7729.

\section{Connaraceae}

Rourea minor (Gaertn.) Leenh.; Sylhet, October 1935, C. S. Purkayastha12446.

\section{Fabaceae}

Abrus precatorius L.; Maulvi Bazar, Sylhet, 11.4.1947, M. M. Srinivasan 22133.

Canavalia ensiformis (L.) DC.; Chhatak, Sylhet, 27.5.1935, G. K. Deka 12814.

Crotalaria juncea L.; Chhatak, Sylhet, 9.2.1941, G. K. Deka 20445; Sylhet, April 1881, G. Mann s.n. 7141.

C. mysorensis Roth; Kalenga, Tarap Reserve, Sylhet, 22.10.1941, R. N. De 20790.

Dalbergia confertiflora Benth.; Ichabil T. E. Sylhet, 28.121914, U. N. Kanjilal 4953; Sylhet, March 1935, C. S. Purkayastha 10983.

D. lanceolaria L. f. Hatikhera; Sylhet, 17.11.1934, U. N. Kanjilal 7816; Longai Reserve, Sylhet, 28.12.1914, U. N. Kanjillal 4950.

D. pinnata (Lour.) Prain; Longai Reserve, Sylhet, March 1935, C. S. Purkayastha 10983.

D. rimosa Roxb.; Tilagarh, Sylhet, 15.11.1932, A. Das 10441; Tilagarh Reserve, Sylhet, 14.12.1938, R. N. De 17601.

Derris cuneifolia Benth.; Lawachara, Sylhet, 20.5.1939, R. N. De 18801; Lawachara, Bhanugach, Sylhet, R. N. De 18732.

D. elegans Benth. var. vestita (Baker) Prain; Lawachara, Bhanugach, Sylhet, 7.7.1939, R. N. De 18665.

Desmodium triflorum (L.) DC.; Fakir Tillah, Chhatak, Sylhet, 18.11.1943, G. K. Deka 22131.

Erythrina fusca Lour.; Chhatak, Sylhet, 27.5.1935, G.K. Deka 12831.

Flemingia macrophylla (Willd.) Merr.; Jintiapur, Sylhet, 7.11.1936, G. K. Deka 16199; Chhatak, Sylhet, 8.2.1914, G. K. Deka 20510.

F. strobilifera (L.) W. T. Aiton; Chittagong Hill Tracts, May 1939, W. J. L Wenger 21512. 
Indigofera arrecta Hochst. ex A. Rich.; Ranpur plantaion, Longai, Sylhet, 19.2.1941, $R$. N. De 20501.

Millettia pachycarpa Benth.; Lawachara, Sylhet, 4.4.1938, R. N. De 16571.

M. pinnata (L.) Panigrahi; Sylhet river bank, 8.8.1921, U.N. Kanjilal 7691.

Ormosia robusta (Kurz) Baker; Raghunandan Reserve, Sylhet, 5.2.1925, U.N. Kanjilal 7838; Patharia forest, Sylhet, March 1886, T. K. Gupta s.n. 8602; Lawachara, Sylhet, April 1937, N.L. Bor 13954.

Pueraria thunbergiana Benth.; Lawachara, Bhanugach, Sylhet, 20.10.1940, R.N. De 19758.

Spatholobus parviflorus (DC.) Kuntze; Longai forest, Sylhet, 16.2.1941, R.N. De 20503; Lawachara, Sylhet, 18.8.1938, R.N. De 18717.

Tadehagi triquetrum (L.) H. Ohashi; Chhatak, Sylhet, 8.2.1940, G. K. Deka 20482; Hatikhera, Sylhet, 17.11.1924, U. N. Kanjilal 7814; Chhatak, Sylhet, 20.11.1943, G. K. Deka 21797.

Uraria crinita (L.) DC.; Lawachaa, Bhanugach, Sylhet, 22.10.1940, R.N. De 19757.

\section{Caesalpiniaceae}

Caesalpinia cucullata Roxb.; Longai forest, Sylhet, 16.2.1941, R.N. De 20393.

Delonix regia (Bojer) Raf.; Road side, Sylhet town, 29.5.1935, G.K. Deka 13412.

Maniltoa polyandra (Roxb.) Harms; Loobah Lake, Sylhet, 9.11.1914, U.N. Kanjilal 4690; Patharia, Sylhet, May 1889, T.K. Gupta s.n. 8823.

Xylia xylocarpa (Roxb.) Taub.; Lawachara, Sylhet, 4.4.1938, R.N. De 16575.

\section{Mimosaceae}

Acacia concinna (Willd.) DC.; Patharia forest, March 1886, G. Mann s.n. 9135.

A. pinnata Link; Chhatak, Sylhet, 8.2.1941, G.K. Deka 20434.

A. polyacantha Willd.; Diopara, Sylhet, October 1937, C.S. Purkayastha 12450.

Albizia lebbek (L.) Benth.; Singla Reserve, Sylhet, 22.12.1914, U.N. Kanjilal 4907.

Archidendron clypearia (Jacq.) I. C. Nielsen; Sylhet, March 1887, T.K. Gupta s.n. 9401; Longai forest, Sylhet, 16.2.1941, R.N. De 20502.

Calliandra umbrosa (Wall.) Benth.; Bholalganj forest, Sylhet, 23.1.1931, G. Ram 1988; Badshaitilla Reserve, Sylhet, 2.12.1924, B. Sen Gupta 7820.

Samanea saman (Jacq.) Merr.; Maulvi Bazar, Sylhet, 11.4.1947, M.M. Srinivasan 21893.

\section{Rosaceae}

Cotoneaster khasiensis Klotz; Sylhet Road, Sylhet, 2.10.1935, A. Das 12280. 


\section{Droseraceae}

Drosera burmanni Vahl; Dinnapur Hill, Sylhet, February 1886, G. Mann s.n.,10413; Chhatak, Sylhet, 8.2.1941, G.K. Deka 20430.

\section{Combretaceae}

Anogeissus sericea Brandis; Longai Reserve, Sylhet, March 1935, C.S. Purkayastha 10989.

Combretum extensum Roxb.; Chhatak, Naya tilla, Sylhet, 8.2.1941, G.K. Deka 20440.

C. pilosum Roxb.; Chhatak, Sylhet, 8.2.1941, G.K. Deka 20438.

Terminalia belirica Wall.; Raghunandan Reserve, Sylhet, 12.4.1947, M.M. Srinivasan 22136.

T. myriocarpa Van Heurck \& Müll.-Arg.; Sylhet, January 1936, C.S. Purkayastha 13434.

\section{Lecythidaceae}

Barringtonia acutangula Gaertn.; Jaintiapur, Sylhet, 7.11.1936, G.K. Deka 16302.

\section{Myrtaceae}

Eucalyptus maculata Hook.; Longai Reserve, Sylhet, 17.2.1941, R.N. De 20560.

Eugenia acuminata Roxb.; Longai Reserve, Sylhet, 27.12.1914, U.N. Kanjilal 4937.

E. aquea Burm. f.; Longai Reserve, Sylhet, 20.6.1925, U.N. Kanjilal 7334.

E. cuneata Wall.; Loobah Lake, Sylhet, 9.11.1914, U.N. Kanjial 4686.

E. cymosa Lam.; Tarap Reserve, Sylhet, March 1935, C.S. Purkayastha 10994; Longai Reserve, Sylhet, 22.2.1915, U.N. Kanjilal 6899; Longai forest, 18.2.1941, R.N. De 20504.

E. grandis Wight; Longai Reserve, Sylhet, March 1835, C.S. Purkayastha 10986; Singla Reserve, Sylhet, 14.3.1921, B. Sen Gupta 7638.

E. jambos L.; Patherkandi, Sylhet, April 1884, T.K. Gupta s.n. 10838.

E. kurzii Duthie ex Kurz; Lawachara, Sylhet, 18.8.1938, R.N. De 18119.

E. mooniana Wight; Longai Reserve, Sylhet, 25.12.1914, U.N. Kanjilal 4927.

\section{Melastomaceae}

Memecylon elegans Kurz; Chhatak, Sylhet, 7.2.1941, G.K. Deka 20453.

Osbeckia nepalensis Hook.; Lawachara, Sylhet, 19.8.1938, R.N. De 19178.

\section{Lythraceae}

Duabanga sonneratioides Buch.-Ham.; Bholaganj, Sylhet, 24.1.1931, R. Sarkar 9009. Lagerstroemia parviflora Roxb.; Patharia forest, Sylhet, March 1886, G. Mann s.n. 11725 . 


\section{Onagraceae}

Ludwigia adscendens (L.) H. Hara; Kamalganj, Sylhet, 14.9.1946, G.K. Deka 22243.

L. octovalvis (Jacq.) P. H. Raven subsp. sessiliflora (Micheli) P. H. Raven; Fakir Tila, Chhatak, Sylhet, 18.11.1943, G.K. Deka 22162.

\section{Passifloraceae}

Modecca cardiophylla Mast.; 44th miles from Sylhet - Shillong Road, 9.8.1944, G.K. Deka 22141.

Passiflora foetida L.; Lawachara, Sylhet, 18.8.1939, R.N. De 19159; Loobah Lake, Sylhet, 8.11.1914, U.N. Kanjilal 4684; Chhatak, Sylhet, 20.11.1943, G.K. Deka 21794.

P. subpeltata Ortega; Lawachara, Sylhet, 18.8.1939, R.N. De 19160.

\section{Cucurbitaceae}

Hodgsonia heteroclita Hook.f. \& Thomson; Shillong-Sylhet Road, 35 miles from Sylhet, 13.4.1947, M.M. Srinivasan 22371.

Melothria heterophylla (Lour.) Cogn.; Lawachara, Sylhet, 18.8.1938, R.N. De 18253.

\section{Aizoaceae}

Mollugo lotoides (Loefl.) Kuntze; Chhatak, bank of Surma river, Sylhet, G K. Deka 20455.

M. pentaphylla L.; Lawachara, Sylhet, 19.8.1938, R.N. De 18041.

48. Araliaceae

Heteropanax fragrans Seem.; Bholaganj, Sylhet, 26.12.1955, G.K. Deka s.n. 39319.

Trevesia palmata Vis.; Dawki, Sylhet, 29.4.1943, G.K. Deka 22019.

49. Alangiaceae

Alangium barbatum Baill. ex Kuntze; Longai Reserve, Sylhet, 24.12.1941, U.N. Kanjilal 4921.

\section{Caprifoliceae}

Viburnum foetidum Wall.; Lawachara, Sylhet, 17.8.1938, R.N. De 18090.

\section{Rubiaceae}

Adenosacme longifolia Wall.; Lawachara, Sylhet, 18.8.1938, G.K. Deka 18101.

Canthium didymum C. F. Gaertn.; Longai Reserve, Sylhet, 24.12.1941, U.N. Kanjilal 4923.

Gardenia coronaria Buch.-Ham.; Bhanugach Reserve, Sylhet, 22.6.1924, B. Sen Gupta 15989; Sylhet, May 1937, N.L. Bor 13928; Kalenga, Sylhet, 21.12.1938, R.N. De 17596. Hedyotis tenelliflora Blume; Jaintiapur, Sylhet, 7.1.1936, G.K. Deka 16463. 
Hymenodictyon excelsum (Roxb.) Wall.; Longai Reserve, Sylhet, 16.2.1941, R.N. De 26521.

H. orixense (Roxb.) Mabb.; Lawachara, Sylhet, 18.8.1938, R.N. De 18054.

Hyptianthera stricta Wall.; Loobah lake, Sylhet, 9.11.1914, U.N. Kanjilal 4688.

Ixora finlaysoniana Wall.; Sylhet, October 1935, C.S. Purkayastha 12437.

I. undulata Roxb.; Raghunandan Reserve, Sylhet, 12.4.1947, M.M. Srinivasan 22137.

Mitragyna diversifolia Havil.; Lawachara, Bhanugach Reserve, Sylhet, 19.12.1938, R.N. De 17726; Longai forest, 16.2.1941, R.N. De 20524.

Nauclea sessilifolia Roxb.; Tarap Reserve, Sylhet, March 1935, C.S. Purkayastha 10984; Tarap Reserve, Sylhet, 13.12.1924, U.N. Kanjilal 7822.

Oldenlandia nudicaulis Roth; Lawachara, Sylhet, 19.8.1938, R.N. De 18834.

Ophiorrhiza oppositifolia Hook.f.; Lawachara, Sylhet, 18.8.1938, R.N. De 18848.

Psydrax glabrum (Blume) Deb \& Dutta; Sylhet, October 1935, C.S. Purkayastha 12434.

Randia dumetorum Lam.; Chhatak, Sylhet, 27.5.1935, G.K. Deka 12809.

R. longiflora Lam.; Habiganj, Sylhet, May 1937, N.L. Bor 13947.

Stephegyne diversifolia Benth. \& Hook.f.; Patharia, Sylhet, May 1884, T. K. Gupta s.n. 13160.

Wendlandia grandis Cowan; Lawachara forest, 23.2.1941, R.N. De 20487.

\section{Asteraceae}

Blumea laciniata DC.; Dawki, Sylhet, 16.4.1940, G.K. Deka 2221; Chhatak, Sylhet, 7.2.1941, G.K. Deka 20426.

Cotula hemisphaerica Wall.; Chhatak, Sylhet, 7.2.1941, G K. Deka 20091.

Elephantopus scaber L.; Sylhet, October 1935, C.S. Purkayastha 13484.

Gamochaeta purpurea (L.) Cabera; Chhatak, Sylhet, 7.2.1941, G K. Deka 20607.

Saussurea affinis Spreng. ex DC.; Chhatak, Sylhet, 9.2.1941, G K. Deka 20449.

Vernonia arborea Buch.-Ham.; Lawachsra forest, Sylhet, 19.8.1938, R.N. De 18817;

Loobah Reserve, Sylhet, 10.11.1914, U.N. Kanjilal 4697.

\section{Campanulaceae}

Lobelia affinis Wall.; Lawachara, Sylhet, 18.8.1938, G K. Deka 18715.

Wahlenbergia gracilis E. Mey.; Chhatak, Sylhet, 9.2.1941, G.K. Deka 22219.

54. Plumbaginaceae

Plumbago indica L.; Moolagul, Sylhet, 26.12.1937, R.N. De 16617.

55. Myrsinaceae

Ardisia floribunda Wall.; Longai forest, Sylhet, 16.2.1941, R.N. De 20566. 
A. thomsonii Mez; Syampara, Chhatak, Sylhet, 20.11.1943, G.K. Deka 21824.

Embelia floribunda Wall.; Lawachara, Sylhet, 4.4.1938, $R$ N. De 16566; Tilagarh forest, Sylhet, 9.4.1947, M M. Srinivasan 22383.

E. gallatlyi King \& Gamble; Mianimukh, Chittagong Hill Tract, March 1880, J.S. Gamble 7906.

E. nutans Wall.; Longai Reserve, Sylhet, 25.12.1914, U.N. Kanjilal 4928.

Maesa indica Wall.; Lawachara, Sylhet, 18.8.1938, R.N. De 18114.

M. ramentacea Wall.; Tarap Reserve, Sylhet, 10.1.1925, U.N. Kanjilal 7823; Lawachara, Sylhet, 23.2.1941, R.N. De 20394; Bhanugach Reserve, Sylhet, 19.2.1938 R.N. De 17599; Sylhet, January 1936, C.S. Purkayastha 13444.

\section{Sapotaceae}

Mimusops elengi L.; Patharkandi, Sylhet, July 1889, T.K. Gupta s.n., 17481.

Sarcosperma arboreum Hook. f.; Loobah lake, Sylhet, 10.11.1914, U N. Kanjilal 4700.

\section{Ebenaceae}

Diospyros montana Roxb.; Syampara, Chhatak, Sylhet, 19.11.1943, G.K. Deka 21823.

D. nigricans Wall.; Singla Reserve, Sylhet, 22.12.1914, U.N. Kanjilal 4912; Sylhet, 5.7.1940, G.K. Deka 19605.

D. pilosula Wall.; Sylhet, 9.11.1914, U N. Kanjilal 4692; Loobah lake, Sylhet, 9.11.1914, U.N. Kanjilal 4692.

D. variegata Kurz; Patharia Reserve, Sylhet, 18.1.1921, U.N. Kanjilal 7646; Balisira Hill, Sylhet, 18.3.1921, B. Sen Gupta 7644.

\section{Symplocaceae}

Symplocos ferruginea Roxb.; Bhanugach Reserve, Sylhet division, 26.2.1925, B. Sen Gupta 7826.

S. racemosa Roxb.; Longai Reserve, Sylhet, 28.12.1914, U.N. Kanjilal 4947; Cheragi, Singla Reserve, Sylhet, 30.11.1924, B. Sen Gupta 7818; Patharia Reserve, Sylhet, March 1886, T.K. Gupta s. n. 17922.

\section{Oleaceae}

Jasminum calycinum Wall. ex Voigt; Sylhet, 2.4.1834. Shyam 22156.

J. coarctatum Roxb.; Lawachara, Sylhet, 23.3.1938, R.N. De 16445.

J. laurifolium Roxb.; Lawachara, Sylhet, 3.4.1938, R.N. De 16568.

J. scandens Vahl; Chhatak, Sylhet, 20.11.1943, G.K. Deka 21796; Tilagarh Reserve, Sylhet, May 1886, G. Mann s.n. 1815.

Linociera grandifolia Elmer; Tharia, Sylhet, 26.12.1955, A. Das 104. 
L. macrophylla Wall.; Bhanugach Reserve, Sylhet, 12.8.1921, U.N. Kanjilal 7692; Singla, Sylhet, 21.12.1914, U.N. Kanjilal 4903.

\section{Apocynaceae}

Aganosma marginata G.Don; Singla, Sylhet, 22.12.1914, U.N. Kanjilal 4909.

Anodendron paniculatum A.DC.; Chhatak, Sylhet, 8.2.1941, G.K. Deka 22307.

Holarrhena pubescens (Buch.-Ham.) Wall. ex G. Don; Patharia forest, Sylhet, March 1885, T.K. Gupta s.n. 18741.

Ichnocarpus frutescens R.Br.; Mollagul Hill, Sylhet, 12.11.1914, U N. Kanjilal 4709; Chhatak, Sylhet, 8.2.1941, G K. Deka 20433; Chhatak, Sylhet, 19.11.1943, G K. Deka 21788.

Rauvolfia serpentina Benth. ex Kurz; Lawachara, Sylhet, 19.5.1939, R.N. De 18802; Plains of Sylhet, April 1877, G. Mann 237.

Wrightia coccinea (Roxb.) Sims; Patherkandi, Sylhet, 12.6.1925, B. Sen Gupta 7835; Bhalukmara, Loobah lake, Sylhet, 8.11.1914, U.N. Kanjilal 4680.

\section{Asclepiadaceae}

Asclepias curassavica L.; Plains of Sylhet, February 1888, G. Mann s.n. 19152.

Calotropis gigantea Dryand; Bholaganj, Sylhet, 17.11.1943, B.B. Shyam 22156.

Cynanchum vincetoxicum Pers.; Deoban, Chatak, Sylhet, 14.6.1940, N.L. Bor 13106.

Gymnema acuminatum Wall.; Mollagul Hill, Sylhet, 12.11.1914, U.N. Kanjilal 4710.

Marsdenia roylei Wight; Longai Reserve, Sylhet, 24.12.1914, U.N. Kanjilal 4920.

\section{Loganiaceae}

Fagraea obovata Wall.; Sylhet, March 1935, C.S. Purkayastha 10996.

Strychnos wallichiana Benth.; Longai Reserve, Sylhet, 27.12.1914, U.N. Kanjilal 4939.

63. Gentianaceae

Crawfurdia fasciculata Wall.; Rangamati, Chittagong, April 1939, W.J.L. Wenger 21518.

Limnanthemum cristatum Griesb.; Sylhet, October 1935, C.S. Purkayastha 12438.

\section{Boraginaceae}

Cordia fragrantissima Kurz; Lawachara, Bhanugach, Sylhet, 20.10.1940, R.N. De 19756.

Ehretia acuminata R.Br.; Chhatak, Sylhet, 27.5.1935, G.K. Deka 12830.

Rotula aquatica Lour.; Loobah lake, Sylhet, 9.11.1914, U.N. Kanjilal 4693.

\section{Convolvulaceae}

Argyreia speciosa Sweet; Kamalganj, Sylhet, 12.11.1937, F. Dastidar 16585. 
Ipomoea obscura Guill.; Chhatak, Sylhet, 2.2.1941, G.K. Deka 20518.

I. uniflora Roem. \& Schult.; Chhatak, Sylhet, 2.11.1943, G.K. Deka 21885.

Operculina turpethum (L.) Silva Manso; Longai forest, Sylhet, 16.2.1941, G.K. Deka 22302.

\section{Scrophulariaceae}

Adenosma capitatum Benth. ex Hance; Fakir Tilla, Chhatak, Sylhet, 17.11.1943, G.K. Deka 23375.

Bonnaya veronicaefolia Spreng.; Chhatak, Sylhet, 7.11.1936, G.K. Deka 16477.

Centranthera grandiflora Benth.; Lawachara, Sylhet, 19.8.1938, R.N. De 18714.

Limnophila hirsuta Benth.; Chhatak, Sylhet, 7.11.1936, G.K. Deka 20518.

Pagesia dianthera (Sw.) Pennell; Chhatak, Sylhet, 9.2.1941, G.K. Deka 22589.

Torenia edentula Griff.; Lawachara, Sylhet, 16.8.1938, G.K. Deka 19325.

Vandellia hirsuta Buch.-Ham. ex Benth.; Lawachara, Sylhet, 18.8.1938, R.N. De 18006.

Veronica anagallis Bong.; Surma river bank, Sylhet, 9.2.1941, G.K. Deka 20516.

\section{Orobanchaceae}

Aeginetia indica L.; Rangamati, Chittagong, May 1939, W.J.L. Wenger 21510.

68. Gesneriaceae

Rhynchoglossum ellipticum A.DC.; Lawachara, Sylhet, 18.8.1938, R.N. De 18004.

R. obliquum Blume; Jaintiapur, Sylhet, 30.10.1935, G.K. Deka 12955.

\section{Bignoniaceae}

Crescentia cujete L.; Sylhet, March 1941, R.N. De 20495; Sylhet, 11.11.1921, U.N. Kanjilal 7675; Sylhet, 11.12.1921, B. Sen Gupta 7695.

Pajanelia rheedii Wight; Sylhet station, February 1886, G. Mann s. n. 21086; Ichabil Tea Garden, Sylhet, 28.12.1914, U.N. Kanjilal 4952.

\section{Acanthaceae}

Daedalacanthus strictus T. Anderson; Longai forest, Sylhet, 19.2.1941, R.N. De 20525.

D. suffruticosus T. Anderson; Loobah Reserve, 10.11.1914, U.N. Kanjilal 4694.

Ebermaiera staurogyne Nees; Lawachara, Sylhet, 20.8.1941, R.N. De 20736.

Hygrophila phlomoides Nees; Fokir Tilla, Chhatak, Sylhet, 17.11.1943, G.K. Deka 22009.

H. polysperma T. Anderson; Chhatak, Sylhet, 7.2.1941, G.K. Deka 20287.

H. salicifolia Nees; Jaintiapur, Sylhet, 30.10.1935, G.K. Deka 12881.

Phlogacanthus asperulus Nees; Jaintiapur, Sylhet, March 1880, G. Mann s.n. 21674.

Staurogyne argentea Wall.; Lawachara, Sylhet, 18.8.1938, G.K. Deka 20277. 
S. thyrsodes Kuntze; Roghunandan Reserve, Sylhet, 12.4.1947, M.M. Srinivasan 23395. Strobilanthes acrocephalus T. Anderson; Rangamati, Chittagong, April 1939, W.J.L. Wenger 21519.

Thunbergia grandiflora Roxb.; Lawachara, Sylhet, 18.8.1938, R.N. De 19052.

T. maculata Lace; Lawachara, Sylhet, 19.8.1938, R.N. De 18460.

71. Verbenaceae

Callicarpa arborea Roxb.; Apha river bank, Sylhet, 11.11.1914, U.N. Kanjilal 4706.

C. longifolia Lam.; Bhanugach, Lawachara, Sylhet, 14.11.1932, Dina Nath 10756.

Clerodendron infortunatum Gaertn.; Teliapara, Sylhet, 12.4.1947, M.M. Srinivasan 22135.

Lantana camara L. var. aculeata (L.) Moldenke; Lawachara, Sylhet, 20.8.1941, R.N. De 20737.

Lippia geminata Kunth; Lookara, Habiganj, Sylhet, 26.2.1941, R.N. De 20390.

Premna latifolia Roxb.; Sautgaon, Sylhet, 10.11.1914, U.N. Kanjilal 4703.

Sphenodesme pentandra Jack; Chhatak, Sylhet, 8.2.1941, G.K. Deka 20437.

S. unguiculata Schauer; Sylhet, 26.12.1937, R.N. De 16014.

Vitex glabrata R.Br.; Raghunandan Reserve, Sylhet, 6.8.1921, U.N. Kanjilal 7687.

V. peduncularis Wall.; Raghunandan Reserve, Sylhet, 6.8.1921, U.N. Kanjilal 7685.

V. pubescens Vahl; Bhanugach Reserve, Sylhet, 13.8.1921, U.N. Kanjilal 7686; Longai Reserve, Sylhet, 27.12.1914, U.N. Kanjilal 4940.

\section{Lamiaceae}

Coleus blumei Benth.; Longai Reserve, Sylhet, 16.2.1941, R.N. De 20604.

Gomphostemma parviflorum Wall.; Singla, Sylhet, 21.12.1914, U.N. Kanjilal 4904.

Leucas linifolia Spreng.; Lawachara, Sylhet, 18.8.1938, R.N. De 19182.

Ocimum basilicum L.; Habiganj, Sylhet, 27.2.1941, R.N. De 20222.

O. canum Sims; Chhatak, Sylhet, 27.5.1935, G. K. Deka 12855.

\section{Amaranthaceae}

Alternanthera achynantha R.Br.; Chhatak, Sylhet, 9.2.1941, G.K. Deka 20286.

A. sessilis R.Br.; Fakir Tilla, Chhatak, Sylhet, 19.11.1943, G.K. Deka 22148.

Cyathula prostrata Blume; Kamalpur, Sylhet, 15.11.1955, R.N. De s.n. 39581.

\section{Chenopodiaceae}

Chenopodium ambrosioides L.; Chhatak, Sylhet, 9.2.1941, G.K. Deka 20464. 


\section{Polygonaceae}

Polygonum viscosum Buch.-Ham. ex D. Don; Longai river bank, Sylhet, 16.2.1941, R.N. De 20529.

\section{Aristolochiaceae}

Aristolochia roxburghiana Klotzsch; Singla, Sylhet, 23.2.1914, U.N. Kanjilal 4913; Rangamati, Chittagong, May 1939, W.J.L. Wenger 21521.

\section{Piperaceae}

Peperomia pellucida Kunth; Lawachara, Sylhet, 18.8.1938, R.N. De 18078.

Piper attenuatum Buch.-Ham. ex Wall.; Chhatak, Sylhet, 7.2.1941, G.K. Deka 20673.

P. griffithii C. DC.; Nayatilla, Chhatak, Sylhet, 8.2.1941, G.K. Deka 20674.

P. longum L.; Sylhet division, March 1935, C.S. Purkayastha 10961.

\section{Myristicaceae}

Myristica amygdalina Wall.; Longai Reserve, Sylhet, 19.11.1934, U.N. Kanjilal 7815.

M. linifolia Roxb.; Guliang village, Sylhet, 11.11.1914, U.N. Kanjilal 4705.

M. longifolia Hook.f. \& Thomson; Near Ranpur plantation, Longai, Sylhet, 19.2.1941, R.N. De 20605.

\section{Lauraceae}

Actinodaphne angustifolia Nees; Lawachara, Sylhet, 19.8.1938, R.N. De 19169.

Alseodaphne owdeni Parker; Longai Reseve, Sylhet, 27.2.1938, R.N. De 16391.

Beilschmiedia brandisii Hook.f.; Longai Reserve, Sylhet, 28.12.1914, U.N. Kanjilal 4948.

Cryptocarya amygdalina Nees; Lawachara, Sylhet, R.N. De 18058, 18.8.1938; Chhatak, Sylhet, 7.2.1941, G.K. Deka 20429.

Litsea laeta Benth. \& Hook.f.; Longai Reserve, Sylhet, 26.12.1914, U.N. Kanjilal 4933.

L. polyantha Juss.; Patharia, Sylhet, May 1887, T.K. Gupta s.n. 249091.

L. sebifera Pers.; Lawachara, Sylhet, 8.7.1939, R.N. De 19193; Sylhet division, October 1935, C.S. Purkayastha 13293.

Machilus bombycina King ex Hook.f.; Barshai Peak, Sylhet, 23.12.1914, U.N. Kanjilal 4916; Barshai Hill, Sylhet, 28.4.1915, U.N. Kanjilal 6898.

M. villosa Hook.f.; Longai forest, Sylhet, 18.2.1941, R.N. De 20531; Chhatak, Sylhet, 8.2.1941, G.K. Deka 20442.

Phoebe lanceolata Nees; Lawachara, Sylhet, 2.4.1938, R.N. De 16567.

\section{Proteaceae}

Helicia robusta Wall.; Raghunandan Reserve, Sylhet, 3.1.1921, B. Sen Gupta 7645. 


\section{Loranthaceae}

Dendrophthoe falcata (L.f.) Ettingsh.; Chhatak, Sylhet, 8.2.1941, G.K. Deka 20198; Sylhet division, March 1935, C.S. Purkayastha 10982.

Helixanthera parasitica Lour.; Longai Reserve, Sylhet, 18.2.1941, R.N. De 20199.

Loranthus globosus Roxb.; Lawachara, Bhanugach, Sylhet, 8.7.1939, R.N. De 18342.

L. gracilifolius Roxb. ex Schult. f.; Maulavi Bazar Range, Sylhet, 1.2.1935, through

D.F.O. 10976.

Macrosolen cochinchinensis (Lour.) Tiegh.; Longai forest, Sylhet, 17.2.1941, R.N. De 20216; Kamalganj Road, Sylhet, 2.4.1940, R.N. De 19267.

Scurrula cordifolia (Wall.) G. Don; Lawachara, Bhanugach Reserve, Sylhet, March 1935, C.S. Purkayastha 10974.

S. parasitica L.; Lawachara, Sylhet, March 1936, C.S. Purkayastha 13463; Longai Reserve, Sylhet, 11.12.1938, R.N. De 17599; Lawachara, Sylhet, 20.8.1941, R.N. De 20798.

Tolypanthus involucratus (Roxb.) Tiegh.; Longai Reserve, Sylhet, 18.2.1941, R.N. De 20200; Longai Reserve, Sylhet, 24.12.1914, U.N. Kanjilal 4925.

Viscum monoicum Roxb.; Longai Reserve, Sylhet, 18.2.1941, R.N. De 20214.

\section{Euphorbiaceae}

Antidesma bunius Spreng.; Raghunandan Reserve, Sylhet, 5.7.1925, U.N. Kanjilal 7829.

A. ghesaembilla Gaertn.; Chhatak, Sylhet, 27.5.1935, G.K. Deka 12823; Santh Gaon, Sylhet, 9.11.1914, U.N. Kanjilal 4685.

A. roxburghii Wall.; Lawachara, Sylhet, 18.8.1938, G.K. Deka 18122.

Aporosa roxburghii Baill.; Tilagarh Reserve, Sylhet, 9.4.1947, M.M. Srinivasan 22100.

Bridelia tomentosa Blume; Tilagarh Reserve, Sylhet, 14.12.1938, R.N. De 17595.

Chaetocarpus castanocarpus Thwaites; Patharia forest, Sylhet, July 1884, T.K. Gupta s.n. 27412; Singla Reserve, Sylhet, 16.1.1932, U.N. Kanjilal 7724; Tilagarh Reserve, Sylhet, 14.12.1938, R.N. De 17594.

Chrozophora rottleri A. Juss.; Chhatak, Sylhet, 27.5.1935, G.K. Deka 12833.

Cyclostemon assamicus Hook. f.; Longai Reserve, Sylhet, 2.3.1915, U.N. Kanjilal 6900.

C. eglandulosus Kurz; Longai Reserve, Sylhet, 2.3.1915, U.N. Kanjilal 6901; Patharia Reserve, Sylhet, 19.3.1932, B. Sen Gupta 16174.

Gelonium multiflorum A. Juss.; Lawachara, Sylhet, 4.4.1938, R.N. De 16569; Raghunandan Reserve, Sylhet, 5.7.1925, U.N. Kanjilal 2830.

Glochidion gamblei Hook. f.; Tarap Reserve, Sylhet, 10.1.1925, U.N. Kanjilal 7824.

G. lanceolarium Voigt; Tarap Reserve, Sylhet, March 1935. C.S. Purkayastha 10990. 
G. sphaerogynum Kurz; Loobah Reserve, Sylhet, 10.11.1914, U.N. Kanjilal 4696.

Mallotus albus Müll.-Arg.; Lawachara, Sylhet, 18.8.1938, G.K. Deka 18124.

M. rapandus Müll.-Arg.; Kamalganj, Sylhet, 27.2.1925, U.N. Kanjilal 7827.

M. roxburghianus Müll.-Arg.; Lawachara, Sylhet, 18.8.1938, G.K. Deka 18121.

Sapium eugerniaefolium Buch.-Ham. ex Wall.; Loobah Reserve, Sylhet, 10.11.1914. U.N. Kanjilal 4695.

Sauropus trinervius Hook. f. \& Thomson ex Müll.-Arg.; Barshai Peak, Sylhet, 23.12.1914, U.N. Kanjilal 4917.

Trewia nudiflora L.; Lawachara, Sylhet, 20.1.1937, through DFO 13599; Circuit house compound, Sylhet, 3.2.1941, R.N. De 20457.

Trigonostemon semperflorens Müll.-Arg.; Chhatak, Sylhet, 9.2.1941, G.K. Deka 20850.

\section{Urticaceae}

Celtis cinnamomea Lindl.; Longai Reserve, Sylhet, 20.3.1921, U.N. Kanjilal 7641.

Gironniera reticulata Thwaites; Badshai Tilla, Sylhet, March 1935, C.S. Purkayastha 10985; Singla Reserve, Sylhet, 16.3.1921, U.N. Kanjilal 7636.

Sarcochlamys pulcherrima Gaudich.; Lawachara, Sylhet, 18.8.1938, R.N. De 18092.

Trema orientalis Blume; Lawachara, Sylhet, 18.8.1938, R. N. De 19189.

\section{Moraceae}

Artocarpus chama Buch.-Ham. ex Wall.; Solgoi tea estate, Sylhet, 29.12.1914, U.N. Kanjilal 4954.

A. lacucha (Roxb.) Buch.-Ham.; Longai Reserve, Sylhet, 27.12.1914, U.N. Kanjilal 4938.

Conocephalus suaveolens Blume; Tilagarh Reserve, Sylhet, 9.4.1947, M.M. Srinivasan 22346.

Ficus fistulosa Reinw. ex Blume; Lawachara, Sylhet, 23.2.1941, R.N. De 20492.

F. heterophylla L. f.; Loobah, Sylhet, 11.11.1914, U.N. Kanjilal 4704.

F. hispida L. f.; Apha river bank, Sylhet, 11.11.1914, U.N. Kanjilal 4707.

F. geniculata Kurz; Sylhet, 6.10.1914, U.N. Kanjilal 4677.

F. infectoria Roxb.; Sylhet station, February 1886, G. Mann s.n. 28015.

F. gibbosa Blume; Maulavi Bazar, Sylhet, February 1886, G. Mann s.n. 27803.

F. obtusifolia Roxb.; Bhallukmara, Tillagarh, Loobah, Sylhet, 8.11.1914, U.N. Kanjilal 4681.

F. pyriformis Hook. \& Arn.; Loobah lake, Sylhet, 9.11.1914, U.N. Kanjilal 4691.

F. retusa L.; Ita Hill, Sylhet, February 1886, G. Mann s n. 27924. 
F. silhetensis Miq.; Sylhet, June 1886, G. Mann s n. 28279.

F. variegata Blume; Sillaoah, Sylhet, June 1886, G. Mann 36a.

Streblus asper Lour.; Plains of Sylhet, April 1877, T.K. Gupta s.n. 27735.

\section{Juglandaceae}

Engelhardtia polystachya Radlk.; Bhanugach Reserve, Sylhet, 12.5.1921, U.N. Kanjilal 7688 .

E. spicata Blume; Sylhet, March 1887, T.K. Gupta s.n. 28944.

\section{Fagaceae}

Castanopsis armata Spach; Sylhet, March 1886, G. Mann s.n. 29568.

C. lanceifolia (Roxb.) Hickel \& A. Camus; Hailpox, Sylhet, August 1935, C.S. Purkayastha 13423.

C. tribuloides A.DC.; Badshai Tilla, Sylhet, 12.3.1921, B C. Sen Gupta 7637.

Quercus fenestrata Roxb.; Sylhet, May 1887, T.K. Gupta s.n. 29096; Patharia, Sylhet, May 1887, T.K. Gupta s.n. 29104.

Q. lappacea Roxb.; Patharia, Sylhet, May 1889, T.K. Gupta s.n. 29174.

Q. semiserrata Roxb.; Sylhet, March 1887, T.K. Gupta s.n. 29158; Longai Reserve, Sylhet, 27.12.1914, U.N. Kanjilal 4936; Longai Reserve, 27.2.1938, Sylhet, R.N. De 16390.

Q. spicata Sm.; Sylhet, March 1935, C.S. Purkayastha 10995; Bhanugach Reserve, Sylhet, 12.5.1921, U.N. Kanjilal 7690.

\section{Salicaceae}

Salix calophylla Wall.; Chhatak, Sylhet, 7.2.1941, G.K. Deka 20436.

S. tetrasperma Roxb.; Longai Reserve, Sylhet, 24.11.1924, B. Sen Gupta 7817.

\section{MONOCOTYLEDONS}

\section{Hydrocharitaceae}

Ottelia alismoides Pers.; Deopara, Sylhet, October 1935, C.S. Purkayastha 12438.

\section{Orchidaceae}

Dendrobium transparens Wall. ex Lindl.; Dawki, Sylhet district,, 30.4.1943, G.K. Deka 22189.

Hetaeria affinis Lindl.; Ranpur plantation, Longai, Sylhet, 19.2.1941, R.N. De 20643; Bathergul, Sylhet, 6.2.1886, G. Mann 23482.

Micropera pallida (Roxb.) Lindl.; Kamalganj, Sylhet, 14.9.1946, G.K. Deka s n. 36095. 
Zeuxine affinis Benth. ex Hook. f.; Rangamati, Chittagong, May 1939, W.J.L. Wenger 23481.

\section{Zingiberaceae}

Caulokaempferia linearis (Wall.) K. Larsen; Dawki, Sylhet, 11.8.1944, G.K. Deka 21872.

Globba racemosa Sm.; Lawachara, Sylhet, 18.8.1938, R.N. De 19619; Lawachara, Sylhet, 15.7.1940, R.N. De 19543.

Zingiber chrysanthum Rosce; Lawachara, Sylhet, 18.8.1938, R.N. De 20319.

\section{Musaceae}

Musa sapientum L.; Lawachera, Sylhet, 18.8.1938, R.N. De17679.

\section{Dioscoreaceae}

Dioscorea decipiens Hook. f.; Bhanugach Reserve, Sylhet, 18.8.1938, R.N. De 18279.

D. hamiltoni Hook. f.; Bhanugach Reserve, Sylhet, 23.10.1940, R.N. De 19750.

D. pentaphylla L.; Sylhet, May 1936, C.S. Purkayastha 12444.

\section{Roxburghiaceae}

Stichoneuron membranaceum Hook. f. \& Thomson; Ranpur plantation, Sylhet, 19.2.1941, R.N. De 20284.

\section{Liliaceae}

Dianella ensifolia (Schlitter) Kitamura; Lawachara plantation, Sylhet, 15.7.1940, R.N. De 19544.

Dracaena terniflora Wall.; Longai Reserve, Sylhet, 25.12.1914, U.N. Kanjilal 4929.

Paris polyphylla Sm.; Rangamati, Chittagong, May 1934, W.J.L. Wenger 21509.

Smilax aspericaulis Wall.; Longai forest, Sylhet, 18.2.1941, R.N. De 20718.

S. prolifera Roxb.; Chhatak, Syampara, Sylhet, 20.11.1943, G.K. Deka 21831; Chhatak, Sylhet, 7.12.1941, G.K. Deka 20427.

\section{Pontederiaceae}

Eichhornia speciosa Kunth; Chhatak, Sylhet, 27.5.1935, G.K. Deka 12867; Sylhet, October 1935, C.S. Purkayastha 12445.

96. Xyridaceae

Xyris pauciflora Willd.; Chhatak, Sylhet, 18.11.1943, G.K. Deka 21850.

\section{Commelinaceae}

Commelina paludosa Blume; Lawachara, Sylhet, 18.8.1938, R.N. De 19623.

Cyanotis cristata (L.) D. Don; Lawachara, Sylhet, 18.8.1938, R.N. De 19622. 
Floscopa scandens Lour.; Fakir tilla, Chhatak, Sylhet, 18.11.1943, G.K. Deka 22420.

Murdannia elata G. Brückn.; Lawachara, Sylhet, 18.8.1938, R.N. De 19627.

M. terminalis (Blume) Raizada; Sylhet, October 1935, C.S. Purkayastha 12308.

M. vaginata G. Brückn.; Bholaganj, Sylhet, 21.8.1935, G.K. Deka 12480.

\section{Arecaceae}

Calamus tenuis Roxb.; Longai forest, Sylhet, 18.2.1941, R.N. De 20709.

C. viminalis Willd.; Chhatak, Sylhet, 7.2.1941, G.K. Deka 20708.

Daemonorops jenkinsianus Mart.; Longai Reserve, Sylhet, 26.12.1914, U.N. Kanjilal 4932.

Licuala peltata Roxb.; Tillagarh Reserve, Sylhet, 28.11.1941, R.N. De 20785.

Plectocomia himalayana Griff.; Loobah Reserve, Sylhet, 10.11.1914, U.N. Kanjilal 4699 .

\section{Araceae}

Colocasia antiquorum Schott; Dawki, Sylhet, 7.8.1940, G.K. Deka 22205.

Homalomena aromatica Schott; Lawachara, Sylhet, 19.8.1939, G.K. Deka 23250.

H. rubescens Kunth; Singla Reserve, Sylhet, 22.12.1914, U.N. Kanjilal 4906.

Lasia heterophylla Schott; Tilagarh Reserve, Sylhet, 19.4.1947, M.M. Srinivasan 22347.

Pistia stratiotes L.; Jaintiapur, Sylhet, 30.10.1935, G.K. Deka 12860.

Pothos scandens L.; Sylhet, October 1935, C.S. Purkayastha 12443.

\section{Cyperaceae}

Carex thomsonii Boott; Plains of Sylhet, April 1887, C.B. Clarke s.n. 31720.

Cyperus brevifolius Hassk.; Sylhet, October 1935, C.S. Purkayastha 12309.

C. iria L.; Sylhet, August 1935, C.S. Purkayastha 12628.

C. sieberianus Spreng.; Sylhet, October1935, C.S. Purkayastha 12286.

C. tenuispica Steud.; Lawachara, Sylhet, August 1935, C.S. Purkayastha 12627.

Eleocharis dulcis Trin. ex Henschel.; Sylhet, August 1935, C.S. Purkayastha 12609.

Fimbristylis complanata Link; Sylhet, October 1935, C.S. Purkayastha 12289.

F. dichotoma (L.) Vahl; Lawachara, Sylhet, August 1935, C.S. Purkayastha 12627.

F. schoenoides Vahl; Fakir tila, Chhatak, Sylhet, 18.11.1943, G.K. Deka 22311.

F. tetragona R. Br.; Shillong-Sylhet Road, 40 mile point, 28.8.1935, G.K. Deka 12362.

Rikliella squarrosus ( L.) J. Raynal; Lawachara Nursery, Sylhet, 14.9.1946, G.K. Deka S.n. 31628.

Rynchospora rubra (Lour.) Makino; Shillong-Sylhet Road, 40 mile point, 29.8.1935, S.R. Sharma 12360. 
Scirpus grossus L. f.; Kamalganj, Sylhet, 14.9.1946, G.K. Deka 22245.

\section{Poaceae}

Acroceras tonkinense (Balansa) C. E. Hubb.; Lawachara plantation, Sylhet, 10.1.1938, G.K. Deka 17683; Lawachara plantation, Sylhet, 18.8.1938, G.K. Deka 17764.

Apluda mutica L.; Companyganj, Sylhet, 26.11.1936, G.K. Deka 21388.

Bambusa nutans Wall. ex Munro; Loobah Reserve, Sylhet, 10.11.1914, U.N. Kanjilal 4701.

B. polymorpha Munro; Protabgarh, Sylhet, December 1889, T.K. Gupta s.n. 32269; Taraf Hill Reserve, Sylhet, 9.7.1937, through D.F.O. 15820 .

B. tulda Roxb.; Barshai Hill, Sylhet, 23.12.1914, U.N. Kanjilal 4915; Jafflong, Sylhet, 6.8.1944, G.K. Deka 22580; Lawachara, Sylhet, 24.11.1941, R.N. De 22578.

Chrysopogon aciculatus Trin.; Lawachara, Sylhet, August 1935, C.S. Purkayastha 12619.

Cyrtococcum accrescens Stapf; Sylhet, October 1935, C.S. Purkayastha 12295.

Dendrocalamus longispathus Kurz; Magura, Sylhet, April 1937, N L. Bor 13915.

Dendrocalamus sp. (Sil Barua); Protabgarh, Sylhet, December 1889, T.K. Gupta s.n. 31883.

Dendrocalamus sp. (Teli Barua); Poldahar, Sylhet, December 1889, T.K. Gupta s.n. 31881.

Dendrocalamus sp. (Baskal); Protabgarh, Sylhet, December 1889, T.K. Gupta s.n. 31887.

Digitaria adscendens (Kunth) Henrard; Sylhet, October 1935, C.S. Purkayastha,12290, 12297; Sylhet, August 1935, C.S. Purkayastha 12607.

D. biformis Willd.; Sylhet, October 1935, C.S. Purkayastha 12296.

D. microbachne Henrard; Lawachara, Sylhet, August 1935, C.S. Purkayastha 12623; Companyganj, Sylhet, 26.11.1936, G.K. Deka 13835.

Echinochloa stagnina P. Beauv.; Lawachara, Sylhet, August 1935, C.S. Purkayastha 12621.

Eleusine indica Gaertn.; Lawachara, Sylhet, August 1935, C. S. Purkayastha 12620; Sylhet, October 1935, C.S. Purkayastha 12288, 12293,12298, 12302.

Eragrostis japonica (Thunb.) Trin.; Rangamati, Chittagong, May 1939, W.J.L Wenger 21513.

E. unioloides Nees ex Steud.; Lawachara, Sylhet, 22.11.1941, R.N. De 20819.

Erianthus fulvus Nees ex Steud.; Jaintiapur, Sylhet, 30.10.1935, R.N. De 12563.

E. longisetosus T. Anderson ex C. B. Clarke; Longai Reserve, Sylhet, March 1935, C.S. Purkayastha 10977; Tillagarh Reserve, Sylhet, 28.2.1941, R.N. De 20110. 
Gigantochloa macrostachya Kurz; Dewadik, Sylhet, December 1889, T.K. Gupta s.n. 32266.

Hygroryza aristata Nees; Sylhet, October 1935, C.S. Purkayastha 12307.

Hymenachne pseudointerrupta C. Muell.; Companyganj, Sylhet, 7.11.1935, G.K. Deka 12545.

Ischaemum goebelii Hack.; Companyganj, Sylhet, 8.11.1935, G.K. Deka 12583.

Leersia hexandra Sw.; Dalairgaon Range, Sylhet, October 1935, C.S. Purkayastha s.n. 32871.

Melocalamus sp.; Protabgarh, Sylhet, December 1883, T.K. Gupta s.n. 32031.

Melocanna bambusoides Trin.; Protabgarh, Sylhet, December 1883, T.K. Gupta s.n. 31907.

Microstegium ciliatum (Trin.) A. Camus; Jaintiapur, Sylhet, 7.11.1936, G.K. Deka 13864.

Neyraudia reynaudiana (Kunth) Keng ex Hitchc.; Longai Reserve, Sylhet, March 1935, C.S. Purkayastha 10978.

Oryza sativa L.; Jaintiapur, Sylhet, 7.11.1936, G.K. Deka 13715; Companyganj, Sylhet, 7.11.1935, G.K. Deka 12536 ; Sylhet, October 1935, C.S. Purkayastha 12301.

Oxytenanthera albo-ciliata Munro; Magura, Sylhet, April 1937, N.L. Bor 13910.

Panicum cambogiense Balansa; Sylhet, October 1935, C.S. Purkayastha 12294, 12300.

P. humedorum Buch.-Ham.; Jaintiapur, Sylhet, 30.10.1935, G.K. Deka 12452.

P. paludosum Roxb.; Hailpur, Sylhet, August 1935, C.S. Purkayastha 12600.

Paspalidium punctatum A. Camus; Sylhet, October 1935, C.S. Purkayastha 12303.

Paspalum conjugatum P. J. Bergius; Sylhet, October 1935, C.S. Purkayastha 12299; Lawachera, Sylhet, August 1935, C.S. Purkayastha 12625.

Phragmites karka Trin. ex Steud.; Companyganj, Sylhet, 26.11.1936, G.K. Deka 12554.

Pseudostachyum polymorphum Munro; Protabgarh, Syhet, December 1889, T.K. Gupta s.n. 31967.

Saccharum procerum Roxb.; Lawachara, Sylhet, 22.11.1941, R.N. De 20800.

S. spontaneum L.; Near Jaintiapur, Sylhet, 30.10.1935, G.K. Deka 12561.

Saccolepis interrupta (Willd.) Stapf; Sylhet division, October 1935, C.S. Purkayastha 12305; Jaintiapur, Sylhet, 30.10.1935, C.S. Purkayastha 12419.

Setaria pallidifusca Stapf \& C. E. Hubb.; Lawachara, Sylhet, 18.8.1938, G.K. Deka 17735; Sylhet division, October 1935, C.S. Purkayastha 12291.

S. palmifolia Stapf; Jaintiapur, Sylhet, 30.10.1935, G.K. Deka 12417. 
Sporobolus indicus R. Br.; Sylhet division, October 1935, C.S. Purkayastha 12285.

Sorghum vulgare Pers.; Lawachara, Sylhet, May 1937, R.N. De 21419.

Themeda villosa Hack.; Companyganj, Sylhet, 8.11.1935, G.K. Deka 12414.

Thysanolaena maxima Kuntze; Maguria, Sylhet, May 1937, N.L. Bor s.n. 33146; Badshai Tilla Reserve, Sylhet, 8.3.1937, AWPO s.n. 33145.

Vetiveria zizanioides Nash; Companyganj, Sylhet, 26.11.1936, G.K. Deka 12574.

\section{Acknowledgements}

The authors are grateful to Dr. M. Sanjappa, Director, Botanical Survey of India, Kolkata and also to Dr. Sandhyajyoti Phukan, Ex Joint Director, Eastern Regional Centre, Shillong, and Dr. A. A. Mao of Arunachal Pradesh Regional Centre, Botanical Survey of India, Itanagar for the facilities.

\section{References}

Bentham, G. and Hooker, J.D.1862-1883. Genera Plantarum. Vols.1-3. Lovell Reeve \& Co., London.

Holmgren P.K., Holmgren, N.H. and Barnett, L.C. 1990. Index Herbariorum, 8th Ed.:179. Koeltz, Konigstein, Germany.

Kanjilal, U.N., Kanjilal, P.C., De, R.N., Das, A. and Bor, N.L. 1934-1940. Flora of Assam. Vols. 1-5. Government of Assam, Shillong.

(Manuscript received on 18 December, 2009; revised on 4 September, 2010) 